\title{
Diseño de una plataforma de acceso abierto para la gestión de información científica
}

\section{Design a platform of open access to scientific information management}

\author{
Alexander José Mackenzie Rivero ${ }^{1, *}$, Joao Merchán Carreño ${ }^{1, \dagger}$, y Karina Virginia Mero Suarez ${ }^{1, \ddagger}$ \\ ${ }^{1}$ Universidad del Sur de Manabí, Ecuador. \\ mackenzie.alexander,edwin.merchan,karina.mero@unesum.edu.ec
}

Fecha de recepción: 9 de agosto de 2016 — Fecha de aceptación: 16 de agosto de 2016

\begin{abstract}
Resumen-El presente estudio se base en la creación de una propuesta de plataforma de acceso abierto para la gestión de información científica en el Instituto nacional de Investigaciones Agrícolas (INIA), lo que permitirá la conformación de una red de conocimiento distribuido especializada en ciencias y tecnologías agrícolas, que garantice seguridad e integridad de la data, niveles de acceso, de fuente abierta, adaptado a las nuevas necesidades, accesible desde cualquier localidad, regido por normativas y estándares internacionales, garantizando el acceso independientemente del sistema operativo con el que cuente el usuario. Utilizando la red de datos WAN instalada en la institución. Logrando que la información del sector agrícola que posee la institución sea puesta a disponibilidad del usuario aprovechando la potencia de difusión del INTERNET. De esta manera la institución cuenta con una herramienta que integra la información del sector agrícola actualmente dispersa por el territorio nacional. Esta investigación está enmarcada dentro de la modalidad de proyecto factible, así mismo se adscribe a un diseño documental y de campo, tipo descriptivo.
\end{abstract}

Palabras Clave-Gestión de información, Acceso abierto, Red de conocimiento, Documentación e información.

Abstract-This present research is based on the creation of a proposed open source platform for the management of scientific information in the National Agricultural Research Institute (INIA), which will allow the creation of a network of distributed knowledge specialized in agricultural science and technology, to ensure security and data integrity, access levels, open source, adapted to new needs, accessible from any location, ruled by international regulations and standards, ensuring access regardless of used operating system. By using the WAN data network installed in the institution. Making the agricultural sector information held by the institution available and accesible with the power of Internet broadcast. Thus the institution has a tool that integrates information currently scattered throughout the country agricultural sector. This research is framed within the modality of feasible project, also is based on a documentary and descriptive design.

Keywords-Information management, Open access, Knowledge network, Documentation and information

\section{INTRODUCCIÓN}

$\mathbf{L}$ a gestión en cualquier ámbito de la vida requiere información como recurso indispensable, aceptándose que sin acceso a ella no se puede aspirar al desarrollo. Para el manejo de la información entendida en el contexto anterior se han establecido diversas alternativas de perfeccionamiento, recolección, difusión, almacenamiento e intercambio. Lo que implica que obtenerla genera unos costos que unidos a su importancia y demanda, le dan un valor que puede ser capitalizado por el que la posea, siendo ella la materia prima para la generación de conocimiento de igual forma incrementa su valor cuanto más es utilizada, en procura de mayor productividad y competitividad, es por esto que la gestión está íntimamente ligado al manejo de la información.

Rodríguez and Muñoz (2010) considera que el motor del desarrollo social y económico está en la información y el conocimiento, a través de la implantación y el uso de las Tecnologías de Información y comunicaciones. En consecuencia,

\footnotetext{
*Master Universitario en Tecnologías de la Información y Telemática Avanzadas

${ }^{\dagger}$ Ingeniero en Sistemas, Magister en Informatica Empresarial.

${ }^{\dagger}$ Ingeniera en Sistemas, Magister en Informatica Empresarial.
}

la información es un activo cuya posesión es intercambiable y compartible sin que el poseedor original tenga que perderla.

El tratamiento de la información hace un mundo complejo en este sentido, en el que intervienen especialistas de muchas disciplinas. El salto cualitativo con respecto al panorama de hace 40-50 años es enorme.

La gestión de información ha sido calificada como la innovación que facilita, con rapidez y oportunidad el manejo de la información. En esta tarea interactúan los recursos informativos, la tecnología para manejarlos y los elementos de gestión. Los sistemas de información surgen como una necesidad de organizar, sistematizar y facilitar la generación, recopilación y difusión de un gran número de recursos de información (RI) en base a criterios de clasificación estandarizados. Las instituciones de investigaciones científicas, para ser organizaciones de éxito en la actualidad como entes generadores de conocimiento, deben utilizar las Tecnologías de Información y Comunicaciones TIC's como herramientas para anticiparse, moverse y adaptarse a los cambios de forma continua, con la tendencia de afianzar más su posicionamiento como instituciones de investigación, tomando en cuenta que la información científica es de importancia estratégica para el desarrollo de toda nación.

La iniciativa de archivos abiertos, ha surgido como una 
alternativa para solucionar muchos de los problemas que hoy enfrentamos cuando trabajamos con sistemas de información y con la información digital contenida en estos; adicionalmente sirve como herramienta de apoyo a los movimientos organizados por los científicos y bibliotecólogos de todo el mundo que buscan liberar los contenidos científico-académicos y dejarlos al alcance de la humanidad (Gómez-Dueñas, 2006). El Movimiento Open Access surge con fuerza en el mundo de la publicación científica. Desde hace algunos años, las iniciativas relacionadas con el Open Access han inundado la bibliografía profesional, tanto de bibliotecarios, documentalistas y profesionales de la información, como de editores comerciales, agentes involucrados en la publicación científica, investigadores, científicos y académicos de todas las ramas del conocimiento.

Por lo tanto, la gestión de información debe incorporar las nuevas tecnologías acompañadas de metodologías para la clasificación de información y estándares de metadatos para su difusión en Internet, de igual forma tiene que estar relacionado con su entorno para recibir, almacenar, procesar datos y distribuir información. "Los artículos que están libremente expuestos en la red, son mucho más citados que aquellos que están escondidos en los arcanos del papel impreso; estos artículos online son citados 4.5 veces más frecuentemente que los que no están en línea" (Gómez-Dueñas, 2006).

El objetivo de este trabajo es describir los aspectos técnicos, y diseño de una plataforma de acceso abierto para la gestión de información científica. Haciendo uso del protocolo de comunicaciones OAI/PMH para el intercambio y acceso abierto a la información científica, así como también la incorporación de estándares internacionales para la clasificación de la información según su área temática mediante el uso del esquema de categorías de materias AGRIS/CARIS uno de los instrumentos metodológicos de trabajo que se usa para el control temático de la información en los Sistemas AGRIS y CARIS, desarrollados por la FAO. Este esquema se debe usar conjuntamente con el tesauro AGROVOC, con el fin de expresar en forma completa y precisa el asunto de que trata un documento o un proyecto de investigación. Esta plataforma permitirá centralizar el acceso a la información científica y garantizar su acceso desde cualquier localidad aprovechando la capacidad de difusión de internet.

Por tal motivo se diseña una plataforma de acceso abierto para la gestión de información científica en el INIA, lo que permitirá la conformación de una red de conocimiento distribuido especializada en ciencias y tecnologías agrícolas.

La investigación consistió en el desarrollo de tres etapas como lo son: Diagnosticar el estado actual referente a los recursos de información generados en las 19 Unidades Ejecutoras que conforman al INIA. Diseñar la estructura del repositorio institucional de acceso abierto que permita cubrir la variedad de recursos de información y finalmente evaluar la plataforma de software libre a proponer como sistema que permita sustentar los servicios a prestar.

\section{DESARROLLO}

\section{Materiales y Métodos}

\section{Diagnóstico}

La información que se muestra en la Tabla 1, corresponde a la primera etapa de la investigación donde se describen y clasifican las actividades realizadas para cubrir la fase de diagnóstico.

El afectado por esta situación es el usuario que no tiene acceso a la información generada en la institución de una manera ágil y eficaz. Entendiéndose por usuario a los investigadores del área agrícola, agricultores, estudiantes y hasta el propio gobierno. Todos ellos tienen necesidades de información de diversa índole para el desempeño más apropiado de sus funciones y que a su vez generan y/o poseen información que de seguro es importante para otros componentes. Al sistematizar los procesos para compartir la información generada, se podrá contar con el patrimonio informacional de la institución sirviendo base para la creación de nuevo conocimiento.

\section{Diseño del Sistema}

En la Tabla 2, se detallan las actividades realizadas como parte del desarrollo de la fase de diseño

El INIA Venezuela, para ser una organización de éxito en la actualidad debe utilizar las tecnologías de Información y Comunicaciones TIC's como herramientas para anticiparse, moverse y adaptarse a los cambios de forma continua, con la tendencia de afianzar más su posicionamiento como institución de investigación, tomando en cuenta que la información agrícola es de importancia estratégica para el desarrollo de Venezuela.

La implantación de una plataforma de acceso abierto, permitirá que la información del sector agrícola con la cual cuenta la institución sea puesta a disposición de la sociedad en tiempo real y de forma dinámica, aprovechando la potencia de difusión del INTERNET, garantizando el procesamiento de los datos, el acceso a la información, una plataforma que soporte los servicios de información y contando con mecanismos de seguridad para resguardar los datos y evitar los accesos no autorizados. De esta manera la institución tendrá un mecanismo que integra la información del sector agrícola actualmente dispersa por el territorio nacional. De igual forma con la normalización, utilización de estándares internacionales para la descripción de recursos electrónicos e implementación de protocolos como el OAI/PMH permite la interconexión con repositorios de otras instituciones relacionadas.

En la Tabla 3, se detallan las actividades realizadas como parte de la evaluación del software disponible para la selección del sistema a utilizar, y en la Tabla 4 los aspectos técnicos en los tres software analizados.

\section{RESULTADOS Y DISCUSIÓN}

Se eligió DSpace (Versión 5X), ya que gracias a su versatilidad es la solución más adecuada cuando se necesita disponer de un repositorio que va a dar soporte a diferentes tipos de documentos y atender a diversas comunidades de usuarios como es el caso de esta investigación. DSpace 
Tabla 1. Diagnostico de la situación actual del INIA referente a sus recursos de información.

\begin{tabular}{|c|c|c|c|c|c|}
\hline \multirow{5}{*}{$\begin{array}{l}\text { Diagnosticar } \\
\text { el estado } \\
\text { actual referente } \\
\text { a los recursos } \\
\text { de información } \\
\text { generados en las } \\
19 \text { Unidades } \\
\text { Ejecutoras que } \\
\text { conforman al } \\
\text { INIA. }\end{array}$} & \multirow{2}{*}{\multicolumn{2}{|c|}{ ESTRATEGIA }} & \multicolumn{3}{|c|}{ MEDICION DE CUMPLIMIENTO } \\
\hline & & & \multicolumn{2}{|c|}{\begin{tabular}{|l|l} 
Objetivo & Actividad \\
\end{tabular}} & Indicador \\
\hline & Organizativas & $\begin{array}{l}\text { 1. Solicitar a } \\
\text { los encargados } \\
\text { de los centros de } \\
\text { documentación y } \\
\text { biblioteca las } \\
\text { estadísticas de } \\
\text { los recursos } \\
\text { documentales } \\
\text { que poseen }\end{array}$ & $\begin{array}{l}\text { 1.Establecer } \\
\text { el número total } \\
\text { de recursos de } \\
\text { información } \\
\text { con los cuales } \\
\text { cuenta la } \\
\text { institución. } \\
\text { 2.Determinar } \\
\text { la carga de } \\
\text { trabajo que } \\
\text { implica la } \\
\text { descripción } \\
\text { y análisis } \\
\text { documental } \\
\text { de cada RI. }\end{array}$ & $\begin{array}{l}\text { 1. Clasificación } \\
\text { de unidades de } \\
\text { información según } \\
\text { el volumen de RI } \\
\text { que posee. }\end{array}$ & $\begin{array}{l}\text { 1. Bibliotecas } \\
\text { jerarquizadas } \\
\text { de acuerdo al } \\
\text { volumen } \\
\text { de información } \\
\text { y área temática. }\end{array}$ \\
\hline & Operativas & $\begin{array}{l}\text { 1. Desarrollo de } \\
\text { un instrumento de } \\
\text { recolección de } \\
\text { información como } \\
\text { herramienta de } \\
\text { apoyo a la actividad } \\
\text { de diagnostico. }\end{array}$ & $\begin{array}{l}\text { 1. Obtener la } \\
\text { información } \\
\text { de forma } \\
\text { estandarizada de } \\
\text { los } 19 \text { centros } \\
\text { de información y } \\
\text { bibliotecas del } \\
\text { INIA. }\end{array}$ & $\begin{array}{l}\text { 1.Distribución } \\
\text { del instrumento } \\
\text { a los centros } \\
\text { del INIA. } \\
\text { 2. Adiestramiento } \\
\text { en la utilización } \\
\text { del instrumento } \\
\text { de recolección } \\
\text { de información }\end{array}$ & $\begin{array}{l}\text { 1.Número de } \\
\text { instrumentos } \\
\text { de recolección } \\
\text { de información } \\
\text { recibidos. } \\
\text { 2.,Cantidad de } \\
\text { personas } \\
\text { adiestradas } \\
\text { en la utilización d } \\
\text { el instrumento de } \\
\text { recolección de } \\
\text { información }\end{array}$ \\
\hline & Funcionales & $\begin{array}{l}\text { 1. Centralización } \\
\text { de los resultados } \\
\text { obtenido } \\
\text { s en el } \\
\text { diagnostico }\end{array}$ & $\begin{array}{l}\text { 1. Sistematización } \\
\text { de los resultados } \\
\text { obtenidos. }\end{array}$ & $\begin{array}{l}\text { 1. Representación, } \\
\text { gráfica, y } \\
\text { cuantificación de los } \\
\text { resultados obtenidos. }\end{array}$ & $\begin{array}{l}\text { 1. Información } \\
\text { clasificada, } \\
\text { información } \\
\text { sistematizada }\end{array}$ \\
\hline
\end{tabular}

Fuente: Elaboración propia.

Tabla 2. Diseñar la estructura del repositorio institucional de acceso abierto a la investigación científica

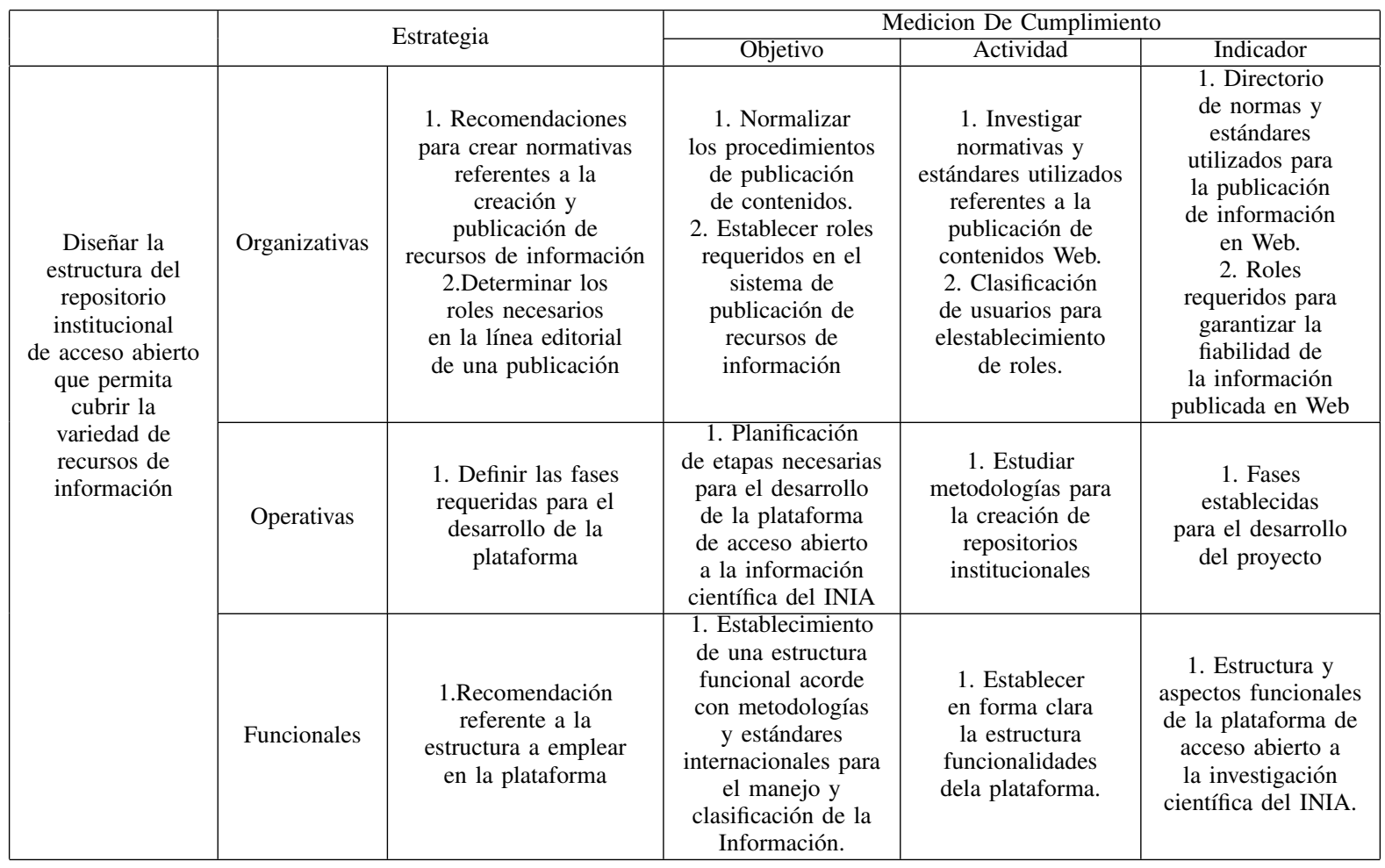

Fuente: Elaboración propia.

acepta documentos en cualquier formato digital incluido, texto, imágenes, video y archivos de audio. Entre estos documentos se encuentran: artículos, reportes técnicos, working papers, papers de conferencia, e-thesis, hojas de datos, librerías de 
Tabla 3. Proceso de evaluación de plataformas de software libre.

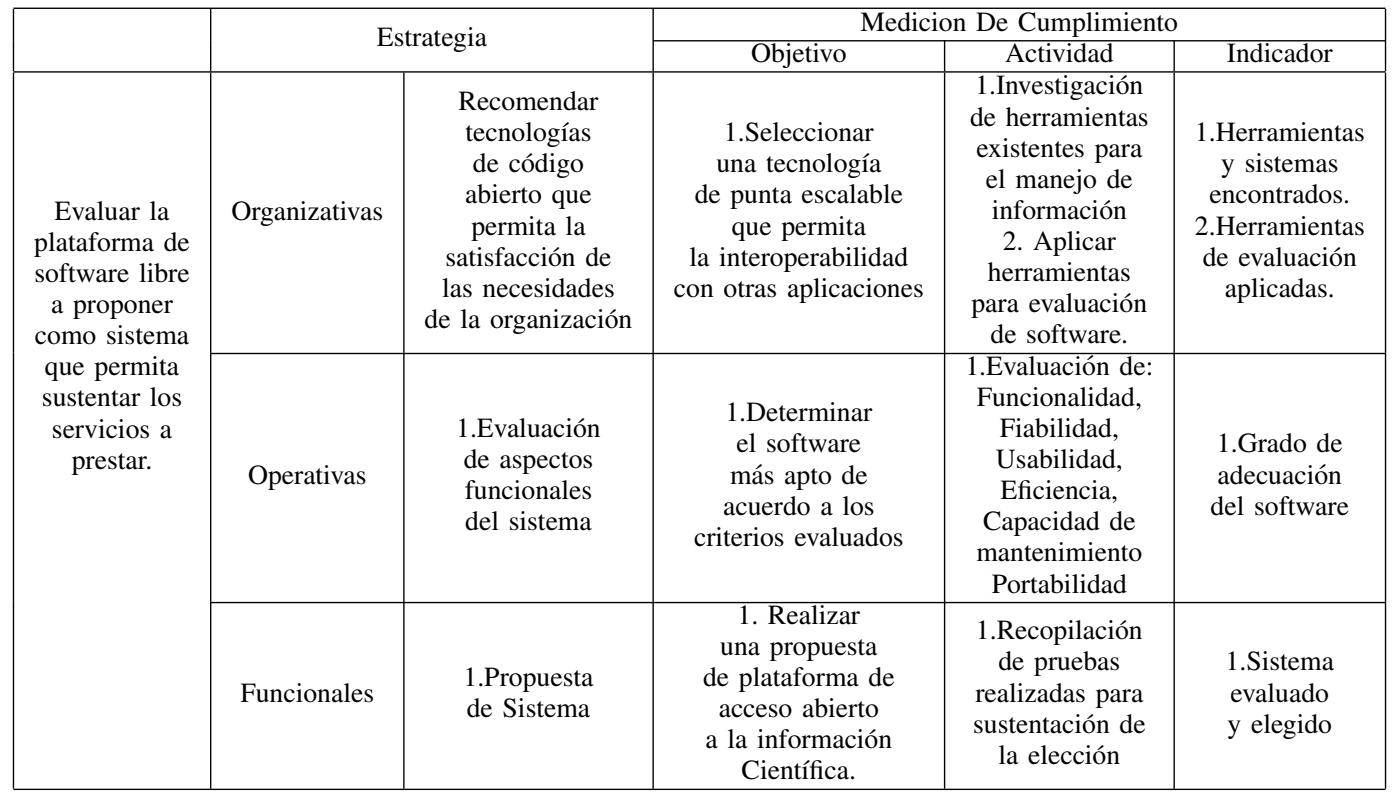

Fuente: Elaboración propia.

Tabla 4. Comparación de aspectos técnicos en los tres software analizados.

\begin{tabular}{|c|c|c|c|}
\hline Características $\backslash$ Nombres & Dspace & Eprints & Fedora \\
\hline Fuente & Open Source & Open Source & Open source \\
\hline Metadatos & Dublin Core, Facilidad para incorporar estándares propios. & Dublin Core & Dublin Core \\
\hline Costo Licencia & $0 \$$ & $0 \$$ & $0 \$$ \\
\hline Sistema Operativo & GNULinux Windows & GNULinux Windows & GNULinux Windows \\
\hline Interoperabilidad & OAIPMH & OAIPMH & OAIPMH \\
\hline Tiempo de Implementación & Medio & Medio & Alto \\
\hline Facilidad de adaptación & Media & Media & Baja \\
\hline Seguridad en Data & Alta & Alta & Alta \\
\hline Soporte técnico & Alto & Medio & Medio \\
\hline Instalaciones a nivel mundial & Alta & Medio & Baja \\
\hline Documentación Técnica disponible & Alta & Alta & Media \\
\hline Interfaz de navegación intuitiva & Alta & Alta & Media \\
\hline
\end{tabular}

Fuente: Elaboración propia.

problemas de prueba, códigos fuente, imágenes, archivos de audio y video, entre otros (Smith et al., 2003).

El usuario es el elemento importante en quien están pensados los procesos de gestión y para quien se desarrollan los sistemas de información. Por lo tanto, se deben considerar los recursos tecnológicos y necesidades de información (Aguilera et al., 2015).

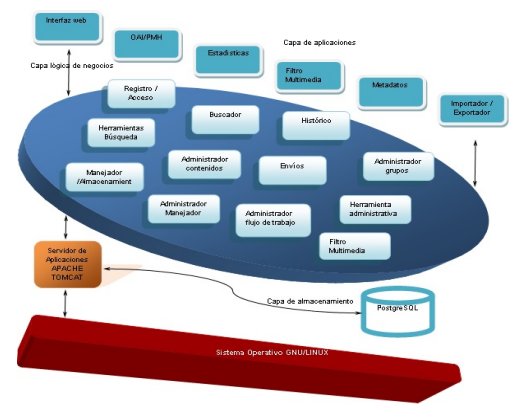

Figura 1. Arquitectura del la plataforma de acceso abierto.

Fuente: Elaboración propia.

En la figura 2 se muestra la interfaz principal de búsqueda desde donde se podrá tener acceso a los contenidos ya sea tanto para la búsqueda y recuperación de información como para la envío de información de manera descentralizada.

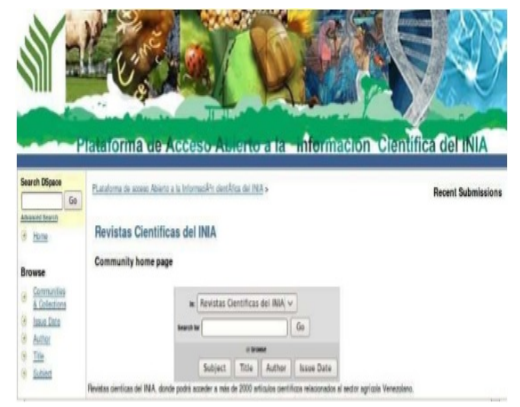

Figura 2. Interfaz de acceso a la plataforma web.

Fuente: Elaboración propia.

La clasificación de los RI está fundamentada en el esquema de categoría de materias AGRIS/CARIS que cubre los tópicos de interés de la FAO, resultando en 17 categorías de materia principales (Comunidades) y 115 categorías de materias segundarias (Colecciones). Se observó que dada la flexibilidad 
de la herramienta se pudo adaptar a una plataforma de acceso abierto especializada en ciencias y tecnologías agrícolas al poder incorporar estándares internacionales para la clasificación de la información facilitando su posterior recuperación.

\section{CONCLUSIONES}

Considerando los resultados obtenidos en el análisis inicial de la situación y el levantamiento de información, se pudo observar que existían serias deficiencias en el manejo de la información del INIA; como consecuencias de la falta de herramientas que permitieran agilizar los procedimientos ejecutados en el área y que dieran soporte fiel a las decisiones en todos los niveles.

En esta perspectiva, la investigación permitió determinar los requerimientos y necesidades de los usuarios, así como la factibilidad para la implementación de la propuesta. Posterior a esto, se evaluaron herramientas existentes con posibilidad de adaptación, detallando la estructura principal de los elementos que componen la plataforma de acceso abierta a la información científica del INIA. La implementación de este sistema generará una serie de beneficios, tales como: rapidez en las transacciones principales, disminución de errores en los cálculos, confiabilidad en los resultados, soporte para la toma de decisiones, ahorro sustancial del tiempo empleado en las actividades de descripción de documentos; además de información oportuna, consistente, y una plataforma abierta para la integración con otras áreas.

La utilización de la plataforma de acceso abierto permitirá el almacenamiento dinámico de las publicaciones, siendo esta actividad realizada por el autor de la obra, lo que permitirá que la documentación se encuentre descrita, clasificada y disponible tan pronto como el autor realice la publicación.

En este sentido, esta plataforma según el análisis realizado demostró ser una aplicación altamente confiable y que ofrece a los usuarios una herramienta eficaz el ingreso y búsqueda de información las 24 horas del día, desde dentro o fuera de la institución. De esta manera, la plataforma informacional se convierte en un medio de información sólido, competente y confiable sustentado en los avances tecnológicos, agilizando el proceso de documentación, y asegurando el acceso oportuno y permanente a la información agrícola, reduciendo las distancias y el tiempo de acceso entre los usuarios y la información

\section{REFERENCIAS BIBLIOGRÁFICAS}

Aguilera, L. R., Rivera, J., Reducindo, I., and Martínez, M. A. O. (2015). Diseño e implementación de un sistema integral para la gestión de archivos de la universidad autónoma de san luis potosí (méxico). Ciencias de la Información, 46(2):9-16.

Gómez-Dueñas, L.-F. (2006). La iniciativa de archivos abiertos (oai): Un nuevo paradigma en la comunicación científica y el intercambio de información. Revista Códice, 2(1):21-48.

Rodríguez, F. M. M. and Muñoz, L. V. A. (2010). Educación y desarrollo socio-económico. Contextos educativos: Revista de educación, (13):83-98.

Smith, M., Barton, M., Bass, M., Branschofsky, M., McClellan, G., Stuve, D., Tansley, R., and Walker, J. H. (2003). Dspace: An open source dynamic digital repository. 